\title{
Area law for black hole entropy in the SU(2) quantum geometry approach
}

\author{
P. Mitra* \\ Saha Institute of Nuclear Physics \\ 1/AF Bidhannagar \\ Calcutta 700064
}

\begin{abstract}
Black hole thermodynamics suggests that a black hole should have an entropy given by a quarter of the area of its horizon. Earlier calculations in U(1) loop quantum gravity have led to a dominant term proportional to the area, but there was a correction involving the logarithm of the area. We find however that $\mathrm{SU}(2)$ loop quantum gravity can provide an entropy that is strictly proportional to the area as expected from black hole thermodynamics.

PACS numbers: 04.60.Pp, 04.70.Dy
\end{abstract}

A black hole at the classical level is a gravitational field, a spacetime with a metric satisfying field equations like those of general relativity. There is a horizon: nothing goes out of this horizon at the classical level. Quantum considerations however indicate the emission of particles at a certain temperature, thereby associating a temperature with the black hole. For a black hole of mass $M$, this temperature is [1]

$$
T=\frac{1}{8 \pi M} .
$$

Theoretical arguments [2] have also assigned an entropy to a black hole, which can be physically understood because the classical one-way horizon restricts the amount of information available to the outside world. This entropy is given by the first law

$$
T d S=d M
$$

of thermodynamics to be

$$
S=4 \pi M^{2}=\frac{A}{4},
$$

where $A=4 \pi(2 M)^{2}$ is the area of the horizon of the black hole. This relation between the area and the entropy of a black hole is also valid for rotating and charged cases.

To understand how the entropy really arises, one would have to identify quantum states, and before that one would need a quantum theory of gravity. No full quantum theory is available, needless to say, but quantum geometry, or loop quantum gravity has made a good beginning [3].

There are gauge fields described by a Chern-Simons theory on the horizon. One has to visualize cross sections of the horizon having punctures. Each puncture has an $\mathrm{SU}(2)$ spin, with quantum numbers $j, m$ associated. Geometrical objects like the area become operators in this approach and have eigenvalues determined by the spin quantum numbers associated with the punctures.

The loop quantum gravity approach to black holes has traditionally fixed the $\mathrm{SU}(2)$ gauge group partially to

\footnotetext{
* parthasarathi.mitra@saha.ac.in
}

$\mathrm{U}(1)$ and therefore considered a U(1) Chern-Simons theory [3]. The level $k$ of the Chern-Simons theory is related to the classical horizon area, to which the quantum eigenvalue is supposed to be close. Each puncture contributes to the quantum eigenvalue of the area an amount proportional to $\sqrt{j(j+1)}$. The spin projection quantum numbers $m$ assigned to punctures have to satisfy a constraint: the sum of $m$ for all punctures must be zero. For a given total area, different configurations of punctures and spins are possible, and these give rise to the number of states for that area, whence an entropy can be derived. Calculations have been extensively made in this approach for the number of quantum states [4 $[6]$. The entropy is proportional to the area for large area, but there is a correction term proportional to the logarithm of the area.

Recently it has appeared to be unnecessary to fix the gauge group, so that the full SU(2) Chern-Simons theory is involved [7]. The constraints are different here, because of the $\mathrm{SU}(2)$ group. Furthermore, the level $k$ of the Chern-Simons theory can be kept finite, free and independent of the area. The aim of the present work was to study the entropy when the area becomes large and see how far the area law given by thermodynamical considerations is obeyed. Calculations can be done both when the level is made large and when it is kept fixed as the area is made large. It was discovered that the entropy is strictly proportional to the horizon area for a fixed level $k$, while the logarithmic term reappears if the level is made large along with the area.

In the $\mathrm{U}(1)$ formulation, the counting is quite straightforward. If one considers $p$ punctures on the horizon of a black hole with $n_{j}$ punctures carrying spin $j$ for different values of $j$, so that $\sum_{j} n_{j}=p$, the number of states is

$$
\frac{\left(\sum_{j} n_{j}\right) !}{\prod_{j} n_{j} !} \prod_{j}(2 j+1)^{n_{j}}
$$

without taking the projection constraint into consideration. For simplicity, let us consider all punctures to have spin $1 / 2$. Then this becomes $2^{p}$. Without the projection constraint, the entropy would be $p \log 2$. But the total projection has to be zero, so $p / 2$ spins have to be up. 
The number of ways for this is $\frac{p !}{(p / 2) !^{2}}$, so the entropy is

$$
\log p !-2 \log (p / 2) ! \approx p \log 2-\frac{1}{2} \log p
$$

by the Stirling approximation. As each puncture contributes equally to the area eigenvalue, $p$ is a measure of the area, so that one gets an area term with a $-\frac{1}{2} \log$ area correction [4].

In the $\mathrm{SU}(2)$ formulation, the number of states for a distribution of spins over punctures arises from properties of $\mathrm{SU}_{q}(2)$ as

$$
N=\frac{2}{k+2} \frac{\left(\sum_{j} n_{j}\right) !}{\prod_{j} n_{j} !} \sum_{a=1}^{k+1} \sin ^{2} \frac{a \pi}{k+2} \prod_{j}\left[\frac{\sin \frac{a \pi(2 j+1)}{k+2}}{\sin \frac{a \pi}{k+2}}\right]^{n_{j}}(6)
$$

[7, 8] 1 . If we take $j_{i}=1 / 2$ for each puncture,

$$
N=\frac{2}{k+2} \sum_{a} \sin ^{2} \frac{a \pi}{k+2}\left[2 \cos \frac{a \pi}{k+2}\right]^{p} .
$$

It is of interest to see what happens if the level $k$ is made large. In this case, the number of terms in the sum over $a$ goes to infinity and it is possible to approximate the sum by an integral. One obtains

$$
N=\frac{2}{k+2} \int_{0}^{k} d a \sin ^{2} \frac{a \pi}{k} 2^{p} \cos ^{p} \frac{a \pi}{k} .
$$

For odd $p$, the integral vanishes, while for even $p$, the range of integration can be halved:

$$
N=\frac{4}{\pi} \int_{0}^{\pi / 2} d x \sin ^{2} x 2^{p} \cos ^{p} x .
$$

This involves a well known integral:

$$
N=\frac{4}{\pi} 2^{p} \frac{\pi}{2} \frac{(p-1) ! !}{(p+2) ! !} .
$$

Now the Stirling approximation for large $n$,

$$
n ! \approx \sqrt{2 \pi n} n^{n} e^{-n}
$$

yields

$$
N \approx 2^{p+3 / 2} p^{-3 / 2} \pi^{-1 / 2} .
$$

This means the entropy is

$$
\log N \approx p \log 2-\frac{3}{2} \log p .
$$

Thus there is an area term and a logarithmic correction with coefficient $-3 / 2$ [7-9].

Next we consider $k$ to be fixed in (7) when $p$ is made large. The argument of the sine/cosine varies from term to term, but the finite sum is dominated by the largest term, which occurs for the largest value of $\left|\cos \frac{a \pi}{k+2}\right|$ in

\footnotetext{
1 The combinatorial factor arises from the choice of the punctures.
}

the available range of $a$. The number of punctures $p$ occurs only in the exponent in $N$ :

$$
N=\frac{4}{k+2} \sin ^{2} \frac{\pi}{k+2}\left[2 \cos \frac{\pi}{k+2}\right]^{p} .
$$

But the area of the horizon is proportional to $p$, hence the entropy

$$
\log N \propto \text { area. }
$$

To see whether the terms with other values of $a$ vitiate this result, one just has to note that for $0<y<x$

$$
\log \left(x^{p}+c y^{p}\right) \approx p \log x+c \exp \left(-p \log \frac{x}{y}\right),
$$

so that the corrections are (a finite number of) exponentially small terms with no $\log p$. This means that there is no logarithmic correction of the usual type when all spins are taken to be $1 / 2$ and $k$ is held constant as $p \rightarrow \infty$.

These arguments can be extended to a general distribution of spins. In this case, we write (6) as

$$
\begin{aligned}
N & =\frac{2}{k+2} \frac{\left(\sum_{j} n_{j}\right) !}{\prod_{j} n_{j} !} \sum_{a=1}^{k+1} \sin ^{2} \frac{a \pi}{k+2} \prod_{j}\left[f_{j}\right]^{n_{j}}, \\
& =\frac{2}{k+2} \frac{\left(\sum_{j} n_{j}\right) !}{\prod_{j} n_{j} !} \sum_{a} \sin ^{2} \frac{a \pi}{k+2} F\left(\cos \frac{a \pi}{k+2}\right),
\end{aligned}
$$

where

$$
f_{j}\left(\cos \frac{a \pi}{k+2}\right) \equiv \frac{\sin \frac{a \pi(2 j+1)}{k+2}}{\sin \frac{a \pi}{k+2}}
$$

and

$$
F\left(\cos \frac{a \pi}{k+2}\right) \equiv \prod_{j}\left[f_{j}\right]^{n_{j}} .
$$

Let us first consider $k$ becoming large, so that the sum over $a$ can be treated as an integral. As $a$ is varied, the integrand

$$
\sin ^{2} \frac{a \pi}{k+2}\left[F\left(\cos \frac{a \pi}{k+2}\right)\right]
$$

attains its maximum when

$$
2 \cot \frac{a \pi}{k+2}=\sin \frac{a \pi}{k+2} \frac{F^{\prime}}{F} .
$$

At this maximum, $a$ satisfies

$$
\left(\frac{a \pi}{k+2}\right)^{2} \approx 2 \frac{F(1)}{F^{\prime}(1)},
$$

which is small because $F^{\prime}$ contains the numbers $n_{j}$ which have to be large for large area. As $\frac{a \pi}{k+2}$ is small, the integrand can be approximated as

$$
\left(\frac{a \pi}{k+2}\right)^{2}\left[F\left(1-\frac{1}{2}\left(\frac{a \pi}{k+2}\right)^{2}\right)\right] .
$$

However, to get an idea of the integral itself, one needs to know the width of the peak, which can be estimated by 
evaluating the second derivative of this expression. The result is

$$
\frac{2 \pi^{2}}{(k+2)^{2}} F-\frac{5 \pi^{4} a^{2}}{(k+2)^{4}} F^{\prime}+\frac{\pi^{6} a^{4}}{(k+2)^{6}} F^{\prime \prime},
$$

which, for large $n_{j}$, simplifies at the maximum to

$$
-4 \frac{\pi^{2}}{(k+2)^{2}} F
$$

Consequently, the width $\sigma$ of the peak is given by

$$
\sigma^{2}=\frac{(k+2)^{2}}{\pi^{2}} \frac{F}{F^{\prime}}
$$

which is essentially the ratio of the maximum to the second derivative and the integral can be approximated by

$$
\frac{2(k+2)}{\sqrt{\pi}}\left(\frac{F}{F^{\prime}}\right)^{3 / 2} F
$$

Now one has to take the combinatorial factor of the punctures into account. The number of states for the distribution can be written as

$$
N=\frac{4}{\sqrt{\pi}} \frac{\left(\sum_{j} n_{j}\right) !}{\prod_{j} n_{j} !}\left(\sum_{j} n_{j} \frac{f_{j}^{\prime}}{f_{j}}\right)^{-3 / 2} \prod_{j}\left[f_{j}\right]^{n_{j}}
$$

by expanding $F$ in terms of the $f_{j}$. To maximize this number, one sets

$$
\delta \log N=0
$$

when the numbers $n_{j}$ of punctures with spin $j$ are varied, subject to the constraint of fixed area

$$
\sum_{j} \sqrt{j(j+1)} \delta n_{j}=0 .
$$

One obtains for large $n_{j}$

$$
\log f_{j}+\log \sum_{k} n_{k}-\log n_{j}-\lambda \sqrt{j(j+1)}=0,
$$

where $\lambda$ is a Lagrange multiplier. One finds then

$$
n_{j}=\left(\sum_{k} n_{k}\right) f_{j} e^{-\lambda \sqrt{j(j+1)}}
$$

whence, for consistency,

$$
\sum_{j=1 / 2}^{k / 2} f_{j} e^{-\lambda \sqrt{j(j+1)}}=1 .
$$

It has to be emphasized that $j$ goes from $\frac{1}{2}$ to $\frac{k}{2}$ for a level $k$, so that for $k \rightarrow \infty$ the upper limit is infinite. In this case, furthermore, $f_{j}$ reduces to $2 j+1$ as $\frac{a}{k+2} \rightarrow 0$ for large area. Now the area

$$
A=\sum_{j} n_{j} \sqrt{j(j+1)}
$$

in appropriate units. Taking the above distribution, one easily sees that

$$
\log N=\lambda A-\frac{3}{2} \log A \quad \text { for } k \rightarrow \infty
$$

as each $n_{j}$ goes like the area for large area. Constant terms are neglected here.

Next, we suppose $k$ to be kept fixed and the area made large. The sum (17) over the finite number of values of $a$ can be considered term by term. For each $a$, the maximization of $N$ with respect to the $n_{j}$ goes through as above. One gets

$$
\log N=\lambda A \text { for finite } k
$$

where $\lambda$ is still determined by (33) with $f_{j}$ evaluated for the $a$ under consideration and the sum restricted to $j \leq k / 2$. Now $\lambda$ depends on $k$ [7] and also on $a$. When the summation of $N$ over $a$ is envisaged, the logic of (16) shows that the highest $\lambda$ dominates and the contributions of values of $a$ giving lower $\lambda$ are exponentially suppressed for large area. In other words, $\lambda$ has to be maximized over $a$, which determines the relevant value(s) of $a$. There is no logarithmic correction in the entropy because the $\left(\frac{F}{F^{\prime}}\right)^{3 / 2}$ factor, which appeared with $a$ taken to be continuous, does not appear in this case of finite $k$ and discrete $a$.

These calculations of the entropy are for the most probable distribution, but the sum over all distributions can also be estimated. There are correction factors proportional to the area from the factorials and from the integrations approximating the sums over $n_{j}$, but these actually cancel out because there is only one constraint, viz., the area constraint (cf. [5]).

In conclusion, if instead of making the level $k$ grow, one holds it fixed and allows the black hole horizon area to become large, the black hole entropy is strictly proportional to the area without a logarithmic correction. If on the contrary the level $k$ is allowed to go to infinity, the usual $\mathrm{SU}(2)$ logarithmic correction with a coefficient $-3 / 2$ is recovered, in accordance with $[7,8]$. What is new is that the possibility, suggested by [7], of keeping the level $k$ of the Chern-Simons theory on the horizon fixed, yields a behaviour of the entropy which is seen to satisfy the thermodynamic area law accurately. The earlier calculations [7, 8] mostly considered $k$ going to infinity, and obtained the log correction which we have also reproduced in that limit. Fixed $k$ was considered in [7] using Laplace transforms and assumptions of analytic continuation, the same logarithmic correction being obtained as for $k$ going to infinity, but they noted that their arguments were less than rigorous. We have explained above why there has to be a difference in the two limits: the factor of $\left(\frac{F}{F^{\prime}}\right)^{3 / 2}$, which is responsible for the logarithmic correction, occurs only for $k \rightarrow \infty$.

Both behaviours are in contrast to the distinctive coefficient $-1 / 2$ for the logarithmic correction in the $\mathrm{U}(1)$ theory. It may be recalled that in the $\mathrm{U}(1)$ counting, there is an extra constraint, the sum over spin projections at all punctures being zero, and that produces a 
$-\frac{1}{2} \log A$ correction. This is seen explicitly in the case when all spins are set equal to $\frac{1}{2}$. More generally, when the number of states is maximized for a distribution of spins and the distribution summed over, the fact that there are two constraints, the area constraint and the spin projection constraint, produces the $-\frac{1}{2} \log A$ correction [5]. This summation does not produce any correction in the $\mathrm{SU}(2)$ case, as pointed out above, because there are no more constraints to be taken care of apart from the area constraint. The $\mathrm{SU}(2)$ constraints are already incorporated into the sum over $a$ in (6). The SU(2) correction arises from two sources: one is the $\sin ^{2}$ factor in (6), and the other is the width of the peak in the sum over $a$. Both of these come into play only in the limit $k \rightarrow \infty$ when the sum is converted to an integral. The $\sin ^{2}$ factor is responsible for a $1 / A$ suppression in $N$ because $\frac{a}{k+2}$ becomes small for large $k$. The width of the peak decreases like $1 / \sqrt{A}$ as the area increases and $k \rightarrow \infty$. For finite $k$, the summation collapses to one or two terms and neither suppression factor appears, so that there is no logarithmic correction.

It must be noted that the $\mathrm{U}(1)$ calculation would produce the logarithmic correction even if $k$ could be kept finite, because the correction comes from a different source there, namely from the spin projection constraint. Thus the possibility of having a strict area law exists only for the $\mathrm{SU}(2)$ theory.

The level $k$ is normally a measure of the classical area of the horizon, while $A$ is the eigenvalue of the area operator. The terms in the entropy therefore relate to the quantum eigenvalue of the area. The logarithmic correction, which arises when $k$ becomes large, might be thought to be caused by a large classical area, being absent when the level $k$ is kept finite and the quantum eigenvalue allowed to soar. However, this limit does not require the classical area to be kept fixed, as quantization ambiguities were exploited [7] to delink the level $k$ from the classical area. One can thus choose to quantize $\mathrm{SU}(2)$ loop quantum gravity in such a way that the entropy grows linearly with the area exactly as predicted by thermodynamical ideas. This is not too dissimilar from the choice made in most versions of loop quantum gravity when the Immirzi parameter [3] is fixed to make the leading contribution to the entropy a quarter of the horizon area instead of being merely proportional to it.

In other words, loop quantum gravity can be tailored to yield the precise area dependence of the entropy predicted from thermodynamical considerations without any logarithmic correction. This is important because no correction has been found to the Hawking temperature [10] which is at the root of the thermodynamical area law.

I thank Amit Ghosh for discussions.
[1] S. W. Hawking, Comm. Math. Phys. 43, 199 (1975)

[2] J. Bekenstein, Phys. Rev. D7, 2333 (1973)

[3] A. Ashtekar, J. Baez and K. Krasnov, Adv. Theor. Math. Phys. 4, 1 (2000)

[4] K. A. Meissner, Class. Quant. Grav. 21, 5245 (2004)

[5] A. Ghosh and P. Mitra, Phys. Letters B616, 114 (2005); A. Ghosh and P. Mitra, Phys. Rev. Letters 102, 141302 (2009)

[6] A. Corichi, J. Diaz-Polo and E. Fernandez-Borja, Phys. Rev. Letters 98, 181301 (2007)
[7] J. Engle, K. Noui and A. Perez, Phys. Rev. Letters 105, 031302 (2010); J. Engle, K. Noui, A. Perez and D. Pranzetti, Phys. Rev. D82, 044050 (2010); A. Perez and D. Pranzetti, arXiv:1011.2961 v3 (2011); J. Engle, K. Noui, A. Perez and D. Pranzetti, JHEP 1105, 016 (2011)

[8] S. Das, R. Kaul and P. Majumdar, Phys. Rev. D63, 044019 (2001)

[9] S. Carlip, Class. Quant. Grav. 17, 4175 (2000)

[10] B. Chatterjee and P. Mitra, Phys. Letters B675, 240 (2009) 